\title{
Combined Toxic Effects of Polar and Nonpolar Chemicals on Human Hepatocytes (HepG2) Cells by Quantitative Property - Activity Relationship Modeling
}

\author{
Ki-Woong Kim', Yong Lim Won', Dong Jin Park', Young Sun Kim', Eun Sil Jin² and Sung Kwang Lee ${ }^{2}$ \\ 'Occupational Safety and Health Research Institute, Korea Occupational Safety and Health Agency, Ulsan, Korea \\ ${ }^{2}$ Department of Chemistry, Hannam University, Daejeon, Korea
}

(Received May 3, 2016; Revised June 13, 2016; Accepted July 26, 2016)

\begin{abstract}
We determined the toxicity of mixtures of ethyl acetate (EA), isopropyl alcohol (IPA), methyl ethyl ketone (MEK), toluene (TOL) and xylene (XYL) with half-maximal effective concentration $\left(\mathrm{EC}_{50}\right)$ values obtained using human hepatocytes cells. According to these data, quantitative property-activity relationships (QPAR) models were successfully proposed to predict the toxicity of mixtures by multiple linear regressions (MLR). The leave-one-out cross validation method was used to find the best subsets of descriptors in the learning methods. Significant differences in physico-chemical properties such as boiling point (BP), specific gravity (SG), Reid vapor pressure (rVP) and flash point (FP) were observed between the single substances and the mixtures. The $\mathrm{EC}_{50}$ of the mixture of EA and IPA was significantly lower than that of contained TOL and XYL. The mixture toxicity was related to the mixing ratio of MEK, TOL and XYL (MLR equation $\mathrm{EC}_{50}=3.3081-2.5018 \times \mathrm{TOL}-3.2595 \times \mathrm{XYL}-12.6596 \times \mathrm{MEK} \times \mathrm{XYL}$ ), as well as to BP, SG, VP and FP (MLR equation $\mathrm{EC}_{50}=1.3424+6.2250 \times \mathrm{FP}-7.1198 \times \mathrm{SG} \times \mathrm{FP}-0.03013 \times$ $\mathrm{rVP} \times \mathrm{FP})$. These results suggest that QPAR-based models could accurately predict the toxicity of polar and nonpolar mixtures used in rotogravure printing industries.
\end{abstract}

Key words: Quantitative property-activity relationship, Rotogravure printing industries, Chemical mixtures, Toxicity

\section{INTRODUCTION}

According to the 2014 Environmental Report (1), over 120,000 different chemicals are dispersed worldwide, and over 2,000 new chemical variations are developed and commercialized each year. More than 40,000 chemicals are distributed in Korea, with over 300 new chemicals introduced to the domestic market annually. Thus, the number of chemicals and their distribution are predicted to increase. The primary reason for the development of novel chemicals and their increased distribution is their unique physical/ chemical properties, which allow their utilization in various fields. Numerous chemicals are used in Korean industry. In

Correspondence to: Ki-Woong Kim, Work Environment Research Bureau, Occupational Safety and Health Research Institute, KOSHA, 400, Jongga-ro, Jung-gu, Ulsan 44429, Korea E-mail: k0810@kosha.net

This is an Open-Access article distributed under the terms of the Creative Commons Attribution Non-Commercial License (http:// creativecommons.org/licenses/by-nc/3.0) which permits unrestricted non-commercial use, distribution, and reproduction in any medium, provided the original work is properly cited. particular, paint manufacturing and printing businesses use high amounts of diverse chemicals. Thus, the risk of health hazards for the employees in these facilities is proportional to the number and amount of chemicals being used. Gravure printing facilities are known to use various chemicals, including benzene (2). These resulted in the regulation of highly toxic chemical use, including benzene. When chemicals exposure at a gravure printing business in Korea was analyzed, the main components in the ink were toluene (CAS No., 108-88-3, TOL), methyl ethyl ketone (CAS No., 78-93-3, MEK), ethyl acetate (CAS No., 141-78-6, EA), isopropyl alcohol (CAS No., 67-63-0, IPA), and xylene (CAS No., 1330-20-7, XYL). Additionally, some inks contained 2-butanol (CAS No., 78-92-2), cyclohexane (CAS No., 110-82-7), and 2-ethoxyethanol (CAS No., 110-80-5) (3). Other studies have also reported that workers in the gravure printing business are exposed to a variety of chemicals, including aromatic hydrocarbons (ethylbenzene, styrene, TOL, XYL, cyclohexane), aliphatic hydrocarbons, ketone bodies, and alcohols. Furthermore, the estimated mixture concentration is higher in gravure printing than in screen or offset printing, indicating that workers in gravure 
printing businesses are likely exposed to higher chemical concentrations (4). The possibility of health hazards increases with exposure to higher concentrations of a single chemical. However, in the case of mixtures, the effect of a single chemical may be altered owing to interactions between chemicals. Kim et al. (5) reported that dimethylformamide (DMF), TOL, and MEK, which are the major components used in synthetic leather manufacturing, significantly differ in their physical/chemical properties as a single substance and a mixture. Thus, exposure levels can vary depending on their handling form (single or mixture). Interestingly, a previous study observed noticeable hemocyte necrosis after injection of a DMF/TOL mixture, but not with a DMF/ MEK mixture (6). This phenomenon can be explained as synergistic hemotoxicity between DMF and TOL, and antagonistic effects between DMF and MEK (7). Importantly, human toxicity prediction studies using mixed substances are ongoing; however, they are insufficient compared to single substance studies. This is most likely because mixed substance studies require more time and have higher costs, since numerous combinations must be used. Furthermore, the number of human and animal studies is restricted owing to research ethics requirements. Therefore, researchers are pursuing studies complementing the aforementioned limitations and producing reliable results. Quantitative property-activity relationships (QPAR) has been used in this field to find key properties (ex. geometric solids, electronic properties, and physical/chemical properties, etc.) without actual experiments or biological information to predict human toxicity, efficacy, and drug reactivity through statistical modeling (8). These studies are primarily performed in the fields of medicine/pharmacology, environmental science, toxicology, and biochemistry, and are gradually expanding to other fields $(9,10)$. Importantly, although the majority of employees handling manufacturing chemicals are exposed to mixed substances, human toxicity studies using mixed substances are limited. Thus, the use of QPAR studies could overcome this challenge. The goal of this study is to compare and analyze the physical/chemical properties and halfmaximal effective concentration $\left(\mathrm{EC}_{50}\right)$ of mixed chemicals handled in gravure printing facilities. We further sought to predict the human toxicity of these mixed chemicals through QPAR analysis.

\section{MATERIALS AND METHODS}

Targeted chemicals and mixing composition. We examined the component ratio of chemicals and mixed substances related to gravure printing facilities through data collection. Data were divided into experimental groups, as shown in Table 1.

Ambient monitoring in workplace. Based on the collected data, the chemicals in the target facilities were con-
Table 1. Classification of experimental groups and mixing ratio of chemicals

\begin{tabular}{ll}
\hline \hline Experimental groups & Mixing ratio (vol/vol) \\
\hline Single & \\
Ethyl acetate (EA) & \\
Isopropyl alcohol (IPA) & \\
Methyl ethyl ketone (MEK) & \\
Toluene (TOL) & \\
Xylene (XYL) & \\
\hline Mixtures & $9: 1$ \\
MEK + EA (G1) & $9: 1$ \\
MEK + IPA (G2) & $8: 1: 1$ \\
MEK + EA + IPA (G3) & $3: 1: 1: 5$ \\
EA + IPA + MEK + TOL (G4) & $3: 1: 1: 5$ \\
EA + IPA + MEK + XYL (G5) & $1: 1: 5$ \\
IPA + MEK + TOL (G6) & $1: 1: 5$ \\
IPA + MEK + XYL (G7) & $3: 1: 1: 2.5: 2.5$ \\
EA + IPA + MEK + TOL + XYL (G8) & \\
\hline
\end{tabular}

Letters in parenthesis represents the groups (G1-G8).

firmed using a manual sampling device (Organic Vapor Monitor 3500, 3M, St. Paul, MN, USA) and active sampling device (LFS-113, Gilian, St. Petersburg, FL, USA) attached to a charcoal tube (Cat. No. 226-01, 226-81, SKC Ltd., Eighty Four, PA, USA). Samples collected by a manual sampling device were subjected to desorption for $1 \mathrm{hr}$ with $2 \mathrm{~mL} \mathrm{CS}$ (1\% butanol). Samples from the charcoal tube collected by the active sampling device were subjected to desorption with $1 \mathrm{~mL} \mathrm{CS} 2$ (1\% butanol). Samples were analyzed according to the official test method 1501 (11) and 2500 (12), as recommended by National Institute of Occupational Safety and Health (NIOSH).

Physico-chemical properties of chemicals. Data on physic-chemical properties of each EA, IPA, MEK, TOL and XYL were obtained from material safety data sheet (MSDS) provided by the Korea Occupational Safety and Health Agency. Physico-chemical properties such as boiling point (BP) (13), specific gravity (SG) (14), Reid vapor pressure (rVP) (15) and flash point (FP) (16) in mixtures were measured.

Experimental determination of half-maximal effective concentrations $\left(E_{50}\right)$. HepG2 cells (human hepatocytes) were acquired from the Korean Cell Line Bank. The cells were cultivated in DMEM (10\% FBS, 100 unit $/ \mathrm{mL}$ penicillin, and $100 \mu \mathrm{g} / \mathrm{mL}$ streptomycin) and MEM badges in a $5 \% \mathrm{CO}_{2}$ atmosphere at $37^{\circ} \mathrm{C}$. They were seeded onto 96-well plates (Corning Inc., Corning, New York, USA) at a concentration of $5 \times 10^{4}$ cells/well and were mixed with single and chemical mixtures after 24 hrs. The cells were transferred into MEM culture medium to be stabilized for $24 \mathrm{hrs}$ at a concentration of $5 \times 10^{4}$ cells/well in a 96-well plate. Samples were grouped as shown in Table 1 and treated 
with the appropriate solutions for 3, 6, 12, and 24 hrs. After culture, the medium was removed and the cells were diluted by $1 / 10$ with the CCK- 8 assay kit (Woongbee, Seoul, Korea) in DMEM culture medium, and then incubated for $1.5 \mathrm{hrs}$ at $37^{\circ} \mathrm{C}$. $\mathrm{EC}_{50}$ values were determined by measuring the absorption of formazan at $450 \mathrm{~nm}$ (7).

Statistical validation of the model. The goodness-offit with the physico-chemical properties or mixing ratio of EA, IPA, MEK, TOL and XYL, as well as the $\mathrm{EC}_{50}$ was carried out using SAS JMP PRO software (ver. 12.0, SAS Institute Inc., Cary, NC, USA). The descriptors were subjected to stepwise selection of multiple linear regression analysis which resulted in descriptors model with least squares fit $\left(\mathrm{R}^{2}\right)$ and leave-one-out cross-validated $\mathrm{R}^{2}$ (LOO $\mathrm{Q}^{2}$ ). The performance of the QPAR regression models has been evaluated by $\mathrm{R}^{2}$, RMSE, and $\mathrm{Q}^{2}$ as follows.

$$
\begin{aligned}
& \mathrm{R}^{2}=1-\frac{\sum\left(\mathrm{y}_{\mathrm{obs}}-\mathrm{y}_{\mathrm{cal}}\right)^{2}}{\sum\left(\mathrm{y}_{\mathrm{obs}}-\mathrm{y}_{\text {mean }}\right)^{2}} \text { for the training set } \\
& \mathrm{Q}^{2}=1-\frac{\sum\left(\mathrm{y}_{\mathrm{obs}}-\mathrm{y}_{\mathrm{pre}}\right)^{2}}{\sum\left(\mathrm{y}_{\mathrm{obs}}-\mathrm{y}_{\text {mean }}\right)^{2}} \text { for the validation set } \\
& \mathrm{RMSE}=\sqrt{\frac{\sum\left(\mathrm{y}_{\mathrm{obs}}-\mathrm{y}_{\mathrm{pre}}\right)^{2}}{N}} \text { for the training set }
\end{aligned}
$$

The QPAR regression model was analyzed by measuring the degree of relevance between the properties used and the estimated $\mathrm{EC}_{50}$ using the linear regression equation (1) below with a training set divided into the pre-set number of folds following a cross-validation test.

$$
y=a_{0}+a_{1} X_{1}+a_{2} X_{2}+\ldots a_{n} X_{n}
$$

Kruskal-Wallis test was performed to assess the differences in physico-chemical properties among the experimental groups, and the QPAR regression model was crossvalidated. All results were presented as mean percentages with standard deviation.

\section{RESULTS}

Identification of chemical substances. Chemical substances handled in the gravure printing facilities were confirmed by ambient monitoring (Table 2). Handling substances differed depending on the printing product; however, the ambient monitoring results showed that EA, MEK, IPA, TOL, and XYL are primarily used.

Composition of physico-chemical properties by experimental groups. The BP, SG, rVP and FP were measured (Table 3). The BP of TOL and XYL alone were $110.0^{\circ} \mathrm{C}$ and $139.2^{\circ} \mathrm{C}$ respectively. These significantly decreased when mixed with EA, MEK, and IPA $(p<0.01)$. The SG of EA alone $(0.90 \mathrm{~g} / \mathrm{mL})$ significantly decreased when mixed with other substances $(p<0.01)$. The rVP of TOL and XYL significantly increased when mixed with EA, IPA, and MEK, as compared to the substance alone $(p<0.01)$. The FP of EA, which has a low FP value $\left(-4.0^{\circ} \mathrm{C}\right)$, was significantly decreased $(p<0.01)$ when mixed with $\operatorname{MEK}\left(-9.0^{\circ} \mathrm{C}\right)$, whereas the FP of MEK was significantly increased when mixed with other substances.

$\boldsymbol{E C}_{50}$ values. The $\mathrm{EC}_{50}$ values for each experimental group were measured using HepG2 cells (Fig. 1). The $\mathrm{EC}_{50}$ values in the single substance treated groups were $4.427 \mu \mathrm{L} /$ $100 \mu \mathrm{L}$ for EA, $4.341 \mu \mathrm{L} / 100 \mu \mathrm{L}$ for IPA, $2.505 \mu \mathrm{L} / 100 \mu \mathrm{L}$ for MEK, respectively, the $\mathrm{EC}_{50}$ values were lower in mixed substances $0.792 \mu \mathrm{L} / 100 \mu \mathrm{L}$ for TOL, and $0.146 \mu \mathrm{L} / 100 \mu \mathrm{L}$ for XYL than single substances, and the differences between the experimental groups were dependent on the $\mathrm{EC}_{50}$ values of the single substances.

Regression model for predicting $\mathrm{EC}_{50}$ in HepG2 cells.

\begin{tabular}{|c|c|c|c|c|c|}
\hline \multirow{2}{*}{ Companies } & \multicolumn{5}{|c|}{ Mean concentrations of ambient chemicals ( $\mathrm{ppm}$ ) } \\
\hline & EA & IPA & MEK & Toluene & Xylene \\
\hline \multicolumn{6}{|c|}{ Rotogravure paint manufacturing companies ( 2 companies) } \\
\hline A & $3.4 \pm 5.1$ & 0.00 & 0.00 & $12.7 \pm 16.1$ & $12.6 \pm 19.1$ \\
\hline $\mathrm{B}$ & $20.6 \pm 19.9$ & $3.2 \pm 2.6$ & $20.6 \pm 33.1$ & $26.5 \pm 32.2$ & $21.5 \pm 21.8$ \\
\hline \multicolumn{6}{|c|}{ Rotogravure printing companies ( 3 companies) } \\
\hline $\mathrm{C}$ & $210.4 \pm 51.1$ & 0.00 & $81.9 \pm 24.7$ & $77.6 \pm 28.7$ & 0.00 \\
\hline $\mathrm{D}$ & $218.1 \pm 291.9$ & $20.9 \pm 51.1$ & $219.4 \pm 307.4$ & $38.9 \pm 55.7$ & $0.7 \pm 2.1$ \\
\hline $\mathrm{E}$ & $140.8 \pm 52.8$ & $4.7 \pm 2.2$ & $178.7 \pm 69.1$ & 0.00 & $3.7 \pm 2.4$ \\
\hline Total & $132.1 \pm 170.5$ & $7.2 \pm 26.5$ & $125.8 \pm 179.1$ & $26.8 \pm 41.5$ & $6.6 \pm 13.6$ \\
\hline TWA & 400 & 200 & 200 & 50 & 100 \\
\hline
\end{tabular}

Regression model by chemical mixing ratios: To predict human toxicity according to the mixing ratio of EA,

Table 2. Identification and ambient levels of chemicals

EA, ethyl acetate; IPA, isopropyl alcohol; MEK, methyl ethyl ketone; TWA, time weighted average. 
Table 3. Results of the physico-chemical properties

\begin{tabular}{lcccc}
\hline \hline \multirow{2}{*}{ Experimental groups } & \multicolumn{3}{c}{ Physico-chemical properties } \\
\cline { 2 - 5 } & $\mathrm{BP}\left({ }^{\circ} \mathrm{C}\right)$ & $\mathrm{SG}(\mathrm{g} / \mathrm{mL})$ & $\mathrm{rVP}(\mathrm{kPa})$ & $\mathrm{FP}\left({ }^{\circ} \mathrm{C}\right)$ \\
\hline Ethyl acetate (EA) & 77.0 & 0.90 & -4.0 \\
Isopropyl alcohol (IPA) & 83.0 & 0.79 & 12.5 & 11.7 \\
Methyl ethyl ketone (MEK) & 80.0 & 0.80 & -9.0 & 4.0 \\
Toluene (TOL) & 111.0 & 0.86 & 21.6 & 29.0 \\
\hline Xylene (XYL) & 139.2 & 0.87 & 2.3 & $-7.37 \pm 0.29$ \\
MEK + EA (G1) & $78.7 \pm 0.1$ & $0.8181 \pm 0.0008$ & $21.23 \pm 0.21$ & $-6.85 \pm 0.29$ \\
MEK + IPA (G2) & $78.5 \pm 0.2$ & $0.8015 \pm 0.0001$ & $21.60 \pm 0.36$ & $-6.53 \pm 0.29$ \\
MEK + EA + IPA (G3) & $77.6 \pm 0.4$ & $0.8110 \pm 0.0001$ & $22.47 \pm 0.38$ & $-2.15 \pm 0.50$ \\
EA + IPA + MEK + TOL (G4) & $83.9 \pm 0.3$ & $0.8615 \pm 0.0001$ & $18.00 \pm 0.44$ & $0.43 \pm 0.01$ \\
EA + IPA + MEK + XYL (G5) & $88.1 \pm 0.3$ & $0.8613 \pm 0.0001$ & $14.63 \pm 0.12$ & $-0.57 \pm 0.50$ \\
IPA + MEK+TOL (G6) & $87.9 \pm 0.1$ & $0.8466 \pm 0.0001$ & $15.93 \pm 0.15$ & $6.31 \pm 0.44$ \\
IPA + MEK + XYL (G7) & $94.4 \pm 0.2$ & $0.8454 \pm 0.0001$ & $11.77 \pm 0.12$ & $-1.01 \pm 0.25$ \\
EA + IPA + MEK + TOL + XYL (G8) & $86.1 \pm 0.2$ & $0.8610 \pm 0.0001$ & $16.20 \pm 0.20$ & 22.40 \\
\hline X (Kuskal Wallis test) & 22.375 & 22.683 & 22.426 & 0.01 \\
$P$ value & 0.01 & 0.01 & 0.01 & 22.406 \\
\hline
\end{tabular}

BP, boiling point; SG, specific gravity; rVP, Reid vapor pressure; FP, flash point.

Letters in parenthesis represents the groups (G1-G8).

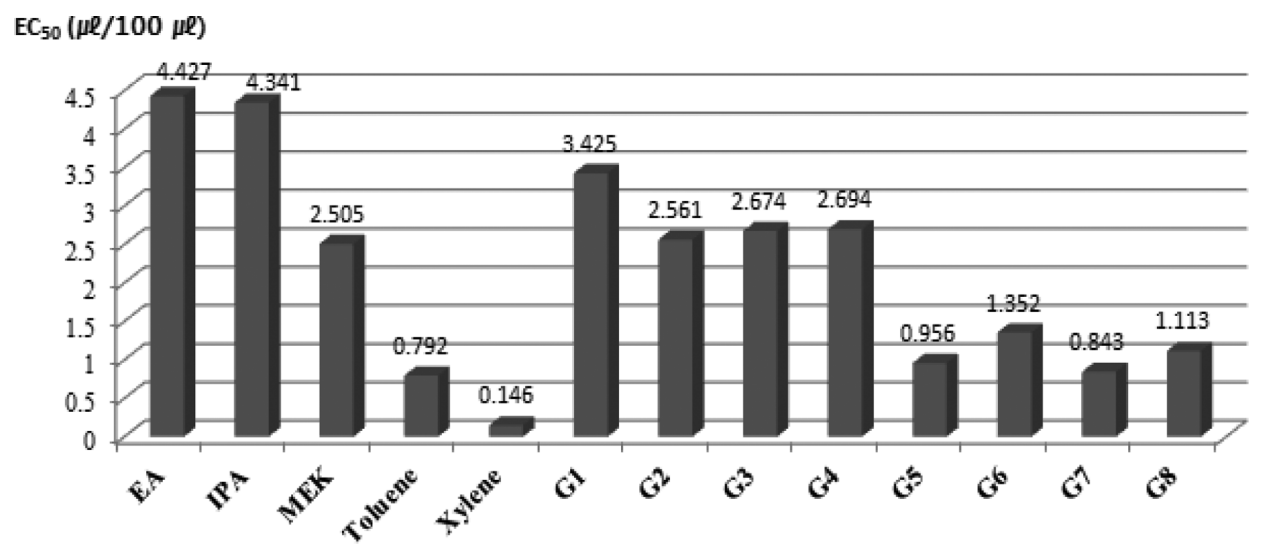

Fig. 1. $E C_{50}(H e p G 2)$ values. $E A$, ethyl acetate; IPA, isopropyl alcohol; $M E K$, methyl ethyl ketone; $G 1, M E K+E A ; G 2, M E K+I P A ; G 3$, MEK + EA + IPA; G4, EA + IPA + MEK + Toluene; G5, EA + IPA + MEK + Xylene; G6, IPA + MEK + Toluene; G7, IPA + MEK + Xylene; G8, EA + $\mathrm{IPA}+\mathrm{MEK}+$ Toluene + Xylene.

Table 4. QPAR model according to chemical mixing ratio by linear regression equation

\begin{tabular}{|c|c|c|c|c|}
\hline \multirow{2}{*}{ Solvent groups } & \multicolumn{2}{|c|}{ Training } & \multirow{2}{*}{$\frac{\mathrm{LOO} C \mathrm{CV}}{\mathrm{Q}^{2}}$} & \multirow{2}{*}{ Linear regression equation } \\
\hline & $\mathrm{R}^{2}$ & RMSE & & \\
\hline MEK, XYL & 0.4487 & 1.1305 & 0.0300 & $\mathrm{y}=2.6437+0.0293 * \mathrm{MEK}-2.9631 * X Y L$ \\
\hline TOL, XYL & 0.7385 & 0.7786 & 0.6006 & $\mathrm{y}=3.2074-2.4922 * \mathrm{TOL}-3.6719 * X Y L$ \\
\hline EA, TOL, XYL & 0.7980 & 0.7214 & 0.4725 & $\mathrm{y}=2.9383+1.2337 * \mathrm{EA}-2.2903 * \mathrm{TOL}-3.4700 * X Y L$ \\
\hline MEK, TOL, XYL & 0.8502 & 0.6211 & 0.6053 & $\mathrm{y}=4.0349-1.4681 * \mathrm{MEK}-3.4798 * \mathrm{TOL}-4.6595 *$ XYL \\
\hline TOL, XYL, MEK * XYL & 0.7870 & 0.7408 & 0.7197 & $\mathrm{y}=3.3081-2.5018 * \mathrm{TOL}-3.2595 * \mathrm{XYL}-12.6596 * \mathrm{MEK} * \mathrm{XYL}$ \\
\hline MEK, TOL, XYL, MEK * XYL & 0.9114 & 0.5006 & 0.6678 & $\begin{aligned} \mathrm{y}= & 4.1971-1.5544 * \mathrm{MEK}-3.5487 * \mathrm{TOL}-4.2526 * \mathrm{XYL} \\
& -14.2728 * \mathrm{MEK} * \mathrm{XYL}\end{aligned}$ \\
\hline EA, MEK, TOL, XYL & 0.8560 & 0.6459 & 0.3670 & $\mathrm{y}=3.8154+0.4561 * \mathrm{EA}-1.2552 * \mathrm{MEK}-3.2620 * \mathrm{TOL}-4.4416 * \mathrm{XYL}$ \\
\hline
\end{tabular}

EA, ethyl acetate; IPA, isopropyl alcohol; MEK, methyl ethyl ketone; TOL, toluene; $X Y L$, xylene; LOO CV, leave-one-out cross-validation; $R^{2}$ and $\mathrm{Q}^{2}$, coefficient; RMSE, root mean square error. 
IPA, MEK, TOL, and XYL, optimized regression models were obtained using a linear regression (Table 4). When we compared the goodness of fit $\left(\mathrm{R}^{2}\right)$ for the training data through regression models by chemical mixing ratios, mixed substances containing TOL and XYL showed goodness of fit between 0.7385 and 0.9114 . To determine the descriptors that affect the model optimization, leave-one-out crossvalidation was performed using the leave-many-out (LMO) method. Our results indicated that $\mathrm{R}^{2}$ ranged from 0.3670 to 0.7197 for mixed substances containing TOL and XYL. The multiple linear regression (MLR) models by mixing ratios of $\mathrm{TOL}+\mathrm{XYL}\left(\mathrm{Q}^{2}=0.6006\right)$ and $\mathrm{MEK}+\mathrm{TOL}+$ $\mathrm{XYL}\left(\mathrm{Q}^{2}=0.6053\right)$ showed $\mathrm{Q}^{2}$ over 0.5 , which is generally considered an acceptance level, but models containing EA were $\mathrm{Q}^{2}<0.5$, which is a standard for validity verification. Furthermore, models containing MEK * XYL as TOL + $\mathrm{XYL}+\mathrm{MEK} * \mathrm{XYL}\left(\mathrm{Q}^{2}=0.7197, \mathrm{y}=3.3081-2.5018 *\right.$ $\mathrm{TOL}-3.2595 * \mathrm{XYL}-12.6596 * \mathrm{MEK} * \mathrm{XYL})$ and MEK + $\mathrm{TOL}+\mathrm{XYL}+\mathrm{MEK} * \mathrm{XYL} \quad\left(\mathrm{Q}^{2}=0.6678, \quad \mathrm{y}=4.1971-\right.$ $1.5544 * \mathrm{MEK}-3.5487 * \mathrm{TOL}-4.2526 * \mathrm{XYL}-14.2728 *$ MEK * XYL) gave cross-validated $\mathrm{Q}^{2}$ value of more than 0.6 and showed that the $\mathrm{EC}_{50}$ value decreased with increasing TOL, XYL and MEK * XYL concentrations. Model containing TOL + XYL + MEK * XYL showed good predictive ability owing to highest cross-validated $\mathrm{Q}^{2}$ value.

Physico-chemical regression model: Using the physico-chemical properties BP, SG, rVP, and FP as descriptors for the linear regression equation, an optimized regression model was obtained (Table 5). Comparing the goodness of fit $\left(\mathrm{R}^{2}\right)$ of the training data in each experimental group by cross-validation showed that, when more than two descriptors were selected, the goodness of fit $\left(\mathrm{R}^{2}\right)$ was 0.5434 0.9623 . Goodness of fit was observed to be high when rVP and FP was combined compared in other physic-chemical property. We conducted the leave-one-out cross validation to determine the descriptors that affect the model optimization. As a results, the models with $\mathrm{rVP} * \mathrm{FP} \mathrm{R} \mathrm{R}^{2}>0.9$ and $\mathrm{Q}^{2}>0.5$. Model containing $\mathrm{FP}+\mathrm{SG} * \mathrm{FP}, \mathrm{rVP} * \mathrm{FP}$ showed good predictive ability owing to highest cross-validated $\mathrm{Q}^{2}$ value and revealed that $\mathrm{EC}_{50}$ value decreased with increasing $\mathrm{SG}$ and $\mathrm{rVP}$ values, and with decreasing $\mathrm{FP}$ values.

\section{DISCUSSION}

Chemical substance development and production is useful in many fields. However, employees who produce these chemicals have a higher risk for health problems, due to chemical exposure (17). Because most of these employees are exposed to mixed compounds (6), it is difficult to elucidate the chemical substances that directly affect health, as well as the interactions between these chemicals. Therefore, we evaluated the chemical substances in mixed compounds and determined which physico-chemical properties have the greatest effect on predicting human toxicity after mixed compound exposure, using single and mixed compounds and $\mathrm{EC}_{50}$ value as descriptors. When we compared the physico-chemical properties and $\mathrm{EC}_{50}$ values of single and mixed compounds, we found that human toxicity due to mixed compounds was dependent on the physico-chemical properties of the single substances. When TOL and XYL were mixed with EA, IPA, and MEK, the BP and FP were lower, whereas the rVP was higher (Table 3). Furthermore, the $\mathrm{EC}_{50}$ values of chemicals with lower toxicity (EA and EPA) decreased (toxicity increased) when they were mixed with other highly toxic chemicals, similar to the changes in physico-chemical properties. Furthermore, the $\mathrm{EC}_{50}$ value of TOL and XYL, which have relatively high toxicity, increased (toxicity decreased) when they were mixed with low toxicity chemicals (Fig. 1). The individual substances composing mixed compounds undergo selective interaction, due to differences in physico-chemical properties and structures. As a result, differences in substance metabolism and toxicity are observed (18). Many studies are being conducted on the absorption and metabolic interactions of mixed compounds in the body; $(19,20)$ however, studies determin-

Table 5. QPAR model according to physico-chemical properties by linear regression equation

\begin{tabular}{|c|c|c|c|c|}
\hline \multirow{2}{*}{$\begin{array}{l}\text { Physico-chemical } \\
\text { properties }\end{array}$} & \multicolumn{2}{|c|}{ Training } & \multirow{2}{*}{$\frac{\mathrm{LOO} C \mathrm{CV}}{\mathrm{Q}^{2}}$} & \multirow{2}{*}{ Linear regression equation } \\
\hline & $\mathrm{R}^{2}$ & RMSE & & \\
\hline BP & 0.4811 & 1.0457 & 0.2215 & $\mathrm{y}=7.0812-0.05513 * \mathrm{BP}$ \\
\hline rVP & 0.4185 & 1.1070 & 0.2654 & $\mathrm{y}=-0.1222+0.1419 * \mathrm{rVP}$ \\
\hline $\mathrm{BP}, \mathrm{FP}$ & 0.5915 & 0.9731 & 0.4664 & $\mathrm{y}=11.0604-0.1006 * \mathrm{BP}+0.08954 * \mathrm{FP}$ \\
\hline rVP, FP & 0.5434 & 1.0288 & 0.4570 & $\mathrm{y}=-2.8460+0.3052 * \mathrm{rVP}+0.1116 * \mathrm{FP}$ \\
\hline $\mathrm{BP}, \mathrm{rVP}, \mathrm{FP}$ & 0.6658 & 0.9277 & 0.1462 & $\mathrm{y}=5.3946-0.06978 * \mathrm{BP}+0.1732 * \mathrm{rVP}+0.1393 * \mathrm{FP}$ \\
\hline $\mathrm{BP} * \mathrm{SG}, \mathrm{BP} * \mathrm{FP}$ & 0.5950 & 0.9687 & 0.3622 & $\mathrm{y}=10.4646-0.1127 * \mathrm{BP} * \mathrm{SG}=0.0007994 * \mathrm{BP} * \mathrm{FP}$ \\
\hline $\mathrm{BP}, \mathrm{SG} * \mathrm{FP}$ & 0.5910 & 0.9734 & 0.4680 & $\mathrm{y}=11.3045-0.1034 * \mathrm{BP}+0.1104 * \mathrm{SG} * \mathrm{FP}$ \\
\hline $\mathrm{FP}, \mathrm{SG} * \mathrm{FP}, \mathrm{rVP} * \mathrm{FP}$ & 0.9479 & 0.3664 & 0.5932 & $\mathrm{y}=1.3424+6.2250 * \mathrm{FP}-7.1198 * \mathrm{SG} * \mathrm{FP}-0.03013 * \mathrm{rVP} * \mathrm{FP}$ \\
\hline $\mathrm{SG}, \mathrm{FP}, \mathrm{SG} * \mathrm{FP}, \mathrm{rVP} * \mathrm{FP}$ & 0.9623 & 0.3303 & 0.5381 & $\mathrm{y}=5.7976-5.2754 * \mathrm{SG}+6.1237 * \mathrm{FP}-7.0023 * \mathrm{SG} * \mathrm{FP}-0.02938 * \mathrm{rVP} * \mathrm{FP}$ \\
\hline
\end{tabular}

BP, boiling point; FP, flash point; rVP, Reid vapor pressure; SG, specific gravity; LOO CV, leave-one-out cross-validation; $\mathrm{R}^{2}$ and $\mathrm{Q}^{2}$, coefficient; RMSE, root mean square error. 
ing toxicity and its mechanism are lacking $(21,22)$. Liira et al. (23) reported that MEK inhibited the metabolism of XYL. Additionally, a study by Tardif et al. (24) reported that mixed compounds with low TOL and XYL concentrations had no effect on substance metabolism at high concentrations. Interestingly, TOL and XYL have metabolic interactions, and inhibited the metabolism of the other. Freundt et al. (25) stated that, after exposure to combined TOL and EA, EA increased the metabolism of TOL, and that this increase was associated with the exposure concentration. Thus, metabolic interactions in mixed compounds are associated with physico-chemical properties and structural similarities, and are dependent on the exposure level (19). Xenobiotics can induce toxicity following absorption by altering pharmacokinetic processes, including absorption, distribution, metabolism, and excretion, depending on their chemical structure and physico-chemical properties. Toxicity can occur at any step in these processes. In contrast, mixed compounds undergo more complicated metabolic processes than single substances, due to the individual pharmacokinetics of the single substances and the pharmacokinetic/pharmacodynamic interactions between individual substances. Human toxicity occurs largely owing to functional inhibition or decreased interaction between metabolic intermediates and adducts, which are generated during the metabolic process, and homeostatic regulatory factors. This is because interactions between components in mixed compounds either increases or inhibits metabolism, thereby antagonizing or synergistically enhancing toxicity (7). When we compared the cell survival rate and $\mathrm{EC}_{50}$ values to examine toxicity between single substances (EA, IPA, MEK, TOL, and XYL) and mixed compounds, there were many differences between the two groups. The cell survival rate was lower with mixed compounds than single substances, and mixing TOL and XYL with EA, IPA, and MEK significantly decreased the cell survival rate. These seem to occur due to changes in physico-chemical properties following interactions between single substances comprising the mixed compound (5). Furthermore, the cell survival rate was positive correlated with the $\mathrm{EC}_{50}$ value in this study. $\mathrm{EC}_{50}$ values for EA and IPA alone were $4.427 \mu \mathrm{L} / 100 \mu \mathrm{L}$ and $4.341 \mu \mathrm{L} /$ $100 \mu \mathrm{L}$, respectively; however, they decreased slightly when mixed with MEK and 4-fold when mixed with TOL and XYL. Interestingly, XYL showed a marked decrease, as compared to TOL. When the toxicity level is compared with the time weight average (TWA) standard of the Ministry of Employment and Labor, TOL toxicity is higher (TOL is $50 \mathrm{ppm}$ and $\mathrm{XYL}$ is $100 \mathrm{ppm}$ ) and $\mathrm{LD}_{50}$ value is lower $(2,600 \mathrm{mg} / \mathrm{kg}$ rat $)$ than XYL $(3,500 \mathrm{mg} / \mathrm{kg}$ rat $)$, indicating that TOL is more toxic. However, Croute et al. (18) reported that TOL cytotoxicity is higher than that of benzene, owing to the side chain attached to the benzene ring and the effect of the lipophilic properties. The $\mathrm{EC}_{50}$ value of $\mathrm{XYL}$ is lower than that of TOL in this study, likely due to the structural properties of the chemicals. In this case, it is presumed to be the effect of the methyl group on the benzene ring. Thus, when the $\mathrm{EC}_{50}$ and $\mathrm{LD}_{50}$ values are used as biological indices to evaluate environmental and human toxicity by chemical substances, evaluation properties should be considered. The toxicity of mixed substances must be predicted using physico-chemical properties, due to the time, cost, and ethical considerations required for experimental research. Thus, in this study, we predicted the toxicity with a QPAR liner regression model using physico-chemical properties and $\mathrm{EC}_{50}$ values as descriptors. As a result, the $\mathrm{Q}^{2}$ value for the TOL + XYL and MEK + TOL + XYL model were 0.6006 and 0.6053 , respectively, exceeding the standard for goodness of fit $\left(Q^{2}=0.5\right)$. The linear regression analysis showed that TOL and XYL reduced the $\mathrm{EC}_{50}$ value among the components of the mixed compounds. In particular, models containing MEK * XYL as TOL + XYL + MEK * XYL $\left(\mathrm{Q}^{2}=\right.$ $0.7197, \mathrm{y}=3.3081-2.5018 * \mathrm{TOL}-3.2595 * \mathrm{XYL}-12.6596 *$ MEK * XYL $)$ and MEK + TOL + XYL + MEK * XYL $\left(Q^{2}=\right.$ $0.6678, \mathrm{y}=4.1971-1.5544 * \mathrm{MEK}-3.5487 * \mathrm{TOL}-4.2526 *$ XYL $-14.2728 *$ MEK * XYL) showed goodness of fit than other solvent models. Furthermore, the results verifying the model optimization according to physico-chemical properties showed that $\mathrm{Q}^{2}$ values for $\mathrm{FP}, \mathrm{SG} \times \mathrm{FP}$ and $\mathrm{rVP} \times \mathrm{FP}$, and a combination of $\mathrm{SG}, \mathrm{FP}, \mathrm{SG} \times \mathrm{FP}$ and $\mathrm{rVP} \times$ FP were 0.5932 and 0.5381 , respectively, exceeding the standard for the goodness of fit $\left(\mathrm{Q}^{2}=0.5\right)$. When we compared the experimental value and $\mathrm{EC}_{50}$ prediction value for descriptors affecting their optimization, the linear regression equation $(y=5.7976-5.2754 \times \mathrm{SG}+6.1237 \times \mathrm{FP}-$ $7.0023 \times \mathrm{SG} \times \mathrm{FP} \times 0.02938 \times \mathrm{rVP} \times \mathrm{FP})$ showed that the $\mathrm{EC}_{50}$ decreased as combinations of $\mathrm{BP} \times \mathrm{SG}, \mathrm{BP}+\mathrm{FP}, \mathrm{SG} \times$ $\mathrm{FP}$ and $\mathrm{rVP} \times \mathrm{FP}$ increased. These results are most likely due to differences in the toxicity expression, which results from changes in physico-chemical properties via interactions between components in the mixed compounds.

In this study, we used physico-chemical properties and $\mathrm{EC}_{50}$ values as descriptors for QPAR modeling to predict the toxicity of mixed compounds containing EA, IPA, MEK, TOL, XYL, which are used in gravure printing. Our results showed that physico-chemical properties and the $\mathrm{EC}_{50}$ values of mixed compounds were dependent on the physicochemical properties of single substances. Toxicity predictions from the linear regression equation using experimental values and prediction $\mathrm{EC}_{50}$ values showed that mixtures containing MEK, TOL and XYL displayed a decreased $\mathrm{EC}_{50}$, but in mixtures containing $\mathrm{MEK} * \mathrm{XYL}$ dramatically decrease $\mathrm{EC}_{50}$ value. Furthermore, either decrease of $\mathrm{SG}$ and $\mathrm{rVP}$ or increase of FP for solvent mixture increase $\mathrm{EC}_{50}$.

\section{ACKNOWLEDGMENTS}

This study was supported by the Intramural Research 
Fund of the Occupational Safety and Health Research Institute of Korea.

\section{REFERENCES}

1. Ministry of Environment (2014) 2014 White paper of environment, Ministry of Environment, Sejong, p. 49.

2. Inoue, O., Seiji, K., Kasahara, M., Nakatsuka, H., Watanabe, T., Yin, S.G., Li, G.L., Jin, C., Cai, S.X. and Wang, X.Z. (1986) Quantitative relation of urinary phenol levels to breathzone benzene concentrations: a factory survey. $\mathrm{Br}$. J. Ind. Med., 43, 692-697.

3. Choi, H.C., Kim, K., An, S.H. and Chung, K.C. (1997) Exposures of organic solvent mixtures to rotogravure printing workers. J. Korean Soc. Occup. Environ. Hyg., 7, 71-85.

4. Kim, Y.M. and Kim, H. (2009) The assessment of health risk and subjective symptoms of printing workers exposed to mixed organic solvents. J. Korean Soc. Occup. Environ. Hyg., 19, 270-279.

5. Kim, K.W., Won, Y.L., Park, D.J., Lee, J.S., Han, I.S. and Lee, S.H. (2014) Changes in physico-chemical properties of single or mixture state of DMF, MEK and toluene in synthetic leather factories. J. Korean Soc. Occup. Environ. Hyg., 24, 238-245.

6. Kim, K.W. and Chung, Y.H. (2013) Hepatotoxicity in rats treated with dimethylformamide or toluene or both. Toxicol. Res., 29, 187-193.

7. Kim, K.W., Won, Y.L., Park, D.J., Kim, D.H. and Song, K.Y. (2014) Comparative study on the $\mathrm{EC}_{50}$ value in single and mixtures of dimethylforamide, methyl ethyl ketone, and toluyene. Toxicol. Res., 30, 199-204.

8. Trichy, M., Trcka, V., Roth, Z. and Krivucova, M. (1985) QSAR analysis and data extrapolationamong mammals in a series of aliphatic alcohols. Environ. Health Perspect., 61, 321-328.

9. Chan, K., Jensen, N. and O’Brien, P.J. (2008) Structure-activity relationships for thiol reactivity and rat or human hepatocyte toxicity induced by substituted $\rho$-benzoquinone compounds. J. Appl. Toxicol., 28, 608-620.

10. Luan, F., Xu, X., Liu, H. and Cordeiro, M.N. (2013) Prediction of the baseline toxicity of non-polar narcotic chemical mixtures by QSAR approach. Chemosphere, 90, 1980-1986.

11. NIOSH (1996) Method 1051 in NIOSH manual of analytical methods (4th edition), National Institute of Occupational Safety and Health, Cincinnati.

12. NIOSH (2003) Method 2500 in NIOSH manual of analytical methods (4th edition), National Institute of Occupational Safety and Health, Cincinnati.

13. Korean Standard (KS) (2007) Determination of boiling temperature for chemical agents (KS M 1071-2), Korean Stan- dard Association, Seoul

14. Korean Standard (KS) (1997) Test methods for density and relative density of chemical products (KS M 0004), Korean Standard Association, Seoul.

15. Korean Standard (KS) (2012) Petroleum products and crude petroleum-Determination of vapour pressure - Reid method (KS M ISO 3007), Korean Standard Association, Seoul.

16. Korean Standard (KS) (2008) Testing methods for flash point of crude oil and petroleum products - Determination of flash point - Tag closed cup method (KS M 2010), Korean Standard Association, Seoul.

17. Kim, K.W. (2015) Effects of styrene-metabolizing enzyme polymorphisms and lifestyle behaviors on blood styrene and urinary metabolite levels in workers chronically exposed to styrene. Toxicol. Res., 31, 355-361.

18. Croute, F., Poinsot, J., Gaubin, Y., Beau, B., Simon, V., Murat, J.C. and Soleilhavoup, J.P. (2002) Volatile organic compounds cytotoxicity and expression of HSP72, HSP90 and GRP78 stress proteins in cultured human cells. Biochim. Biophys. Acta, 1591, 147-155.

19. Ikeda, M. (1995) Exposure to complex mixtures: implications for biological monitoring. Toxicol. Lett., 77, 85-91.

20. Zepeda, A., Twxier, A.C., Razo-Flores, E. and Gomez, J. (2006) Kinetic and metabolic study of benzene, toluene and m-xylene in nitrifying batch cultures. Water Res., 40, 16431649.

21. Chen, C.S., Hseu, Y.C., Liang, S.H., Kuo, J.Y. and Chen, S.C. (2008) Assessment of genotoxicity of methyl-tert-butyl ether, benzene, toluene, ethylbenzene, and xylene to human lymphocytes using comet assay. J. Hazard. Mater., 153, 351-356.

22. Haro-Garcia, L.C., Juarez-Perez, C.A., Aguilar-Madrid, G., Velez-Zamora, N.M., Munoz-Navarro, S., Chaon-Salinas, R., Gonzalez-Bonilla, C.R., Iturbe-Haro, C.R., Estrada-Garcia, I. and Borja-Aburto, V.H. (2012) Production of IL-10, TNF and IL-12 by peripheral blood mononuclear cells in Mexican workers exposed to a mixture of benzene-toluene-xylene. Arch. Med. Res., 43, 51-57.

23. Liira, J., Riihimaki, V., Engstrom, K. and Pfaffli, R. (1988) Coexposure of man to $\mathrm{m}$-xylene and methyl ethyl ketone. Scand. J. Work Environ. Health, 14, 322-327.

24. Tradif, R., Lapare, S., Plaa, G.L. and Brodeur, J. (1991) Effects of simultaneous exposure to toluene and xylene on their respective biological exposure indices in humans. Int. Arch. Occup. Environ. Health, 63, 279-284.

25. Freundt, K.J., Romer, K.G. and Federsel, R.J. (1989) Decrease of inhaled toluene, ethyl benzene, m-xylene, or mesitylene in rat blood after combined exposure to ethyl acetate. Bull. Environ. Contam. Toxicol., 42, 495-498. 